\title{
非扭曲异宿环分支 *
}

田清平 朱德明

(华东师范大学数学系, 上海 200062)

\begin{abstract}
摘要孝虑高维系统非扭曲细异宿环分支, 给出了1-同宿轨道和 1-周期轨道的存 在性和存在域, 并得到了2-重周期轨道的分支曲面。最后,这些分支结果被应用于平 面系统细异宿环, 获得了新的有趣的结论。
\end{abstract}

\section{关键词 异宿轨道 同宿轨道 周期轨道 内稳定性}

近 20 年来, 已经得到了很多关于连接两个双曲鞍点 $p_{i}(i=1,2)$ 的异宿环分支的结果. 文 献 [1]对平面系统给出了下面的结果: 设 $\Gamma$ 是平面系统的一个异宿环, 扰动是 $C^{\infty}$ 的, $r_{10} r_{20} \neq 1$ 且 $r_{10} \neq 1, r_{20} \neq 1$, 则 $\Gamma$ 至多可分支出两个极限环, 其中 $r_{i 0}=\lambda_{1}^{i} / \rho_{1}^{i}, i=1,2, \lambda_{1}^{i}>0$ 和 $-\rho_{1}^{i}<0$ 为未扰动系统在 $p_{i}$ 处的特征值. 文献 [2]证明了当未扰动和扰动系统均为 $C^{\infty}$ 且 $r_{10} r_{20} \neq 1$ 时, $\Gamma$ 至多能分支出两个极限环. 在 $r_{10} r_{20}=1$ 的情况下, 文献 [3]获得了异宿环的稳定性条 件. 特别当 $r_{10}=r_{20}=1$ 时, 文献 [4]亦给出了异宿环稳定性准则的表达式.

近几年来, 已有许多论文致力于研究高维系统的异宿分支 (参见文献 $[5 \sim 10]$ 及其所列文 献). 应用由文献 [11]首先引人的局部坐标系, 文献 $[10]$ 研究了 1 -同宿分支曲面的存在性及 其与 1 - 异宿分支曲面的相切性, 并给出了 1-周期轨道的存在域. 此处,一个 1 -同宿 (或 1 -周 期, 1 - 异宿) 轨道是一个在原异宿环邻域具缠绕数 1 的同宿 (或周期, 异宿)轨道. 本文将文献 $[10]$ 的结果推广到 $\rho_{1}^{1} / \lambda_{1}^{1}=\lambda_{1}^{2} / \rho_{1}^{2}$ 的情况, 其中 $\lambda_{1}^{i},-\rho_{1}^{i}(i=1,2)$ 为未扰动系统在 $p_{i}$ 处的主特 征值, 分别给出了在 $\Gamma$ 邻近存在 2-重 1 - 周期轨道, 两个 1 - 周期轨道, 一个简单 1 - 周期轨道, 和 一个 1 - 周期轨道与一个 1 - 同宿轨道的条件, 并得到了相应的分支曲面. 最后, 上述结果被应 用于平面异宿环, 获得了相应的分支图. 特别地, 证明了当 $r_{10} r_{20}=1$ 而 $\left(\lambda_{1}^{2} / \rho_{1}^{1}\right)^{\beta-1} \sigma_{1} \sigma_{2}^{\beta} \neq 1$ 时, $\Gamma=\Gamma_{1} \cup \Gamma_{2}$ 仍至多能分支出两个极限环, 其中 $\beta=\rho_{1}^{1} / \lambda_{1}^{1}, \ln \sigma_{i}$ 为发散量沿异宿轨 $\Gamma_{i}$ 的积分.

\section{1 假设和局部坐标}

考虑 $C^{r}$ 系统及其扰动

$$
\begin{gathered}
\dot{z}=f(z)+g(z, \mu), \\
\dot{z}=f(z),
\end{gathered}
$$

其中 $r \geqslant 3, z \in \mathbb{R}^{m+n}, \mu \in \mathrm{R}^{k}, k \geqslant 2,0 \leqslant|\mu| \ll 1, f\left(p_{i}\right)=0, g(z, 0)=0$. 为简洁计, 设 $g\left(p_{i}\right.$, $\mu)=0, i=1,2$. 
假设系统 (1.2) 有一个异宿环, $\Gamma=\Gamma_{1} \cup \Gamma_{2}$, 其中 $\Gamma_{i}=\left\{z=r_{i}(t): t \in \mathbb{R}\right\}, r_{1}(-\infty)=$ $r_{2}(+\infty)=p_{1}, r_{1}(+\infty)=r_{2}(-\infty)=p_{2} . D_{z} f\left(p_{i}\right)$ 的特征值 $-\rho_{m}^{i}, \cdots,-\rho_{1}^{i}, \lambda_{1}^{i}, \cdots, \lambda_{n}^{i}$ 满足

$$
-\operatorname{Re} \rho_{m}^{i} \leqslant \cdots \leqslant-\operatorname{Re} \rho_{2}^{i}<-\rho_{0}^{i}<-\rho_{1}^{i}<0<\lambda_{1}^{i}<\lambda_{0}^{i}<\operatorname{Re} \lambda_{2}^{i} \leqslant \cdots \leqslant \operatorname{Re} \lambda_{n}^{i},
$$

其中 $\rho_{0}^{i}$ 和 $\lambda_{0}^{i}$ 为正常数. 进一步设

（H1）(通有假设 1) 记 $W_{i}^{s}, W_{i}^{u}$ 为 $p_{i}$ 的稳定和不稳定流形, $p_{3} \equiv p_{1}, e_{i}^{ \pm}=\lim _{t \rightarrow \pm \infty} \dot{r}_{i}(-t) /$ $\left|\dot{r}_{i}(-t)\right|$, 则 $e_{i}^{+} \in T_{p_{i}} W_{i}^{u}$ 和 $e_{i}^{-} \in T_{p_{i+1}} W_{i+1}^{s}$ 分别是相应于 $\lambda_{1}^{i}$ 和 $-\rho_{1}^{i+1}$ 的单位特征向量.

（H2）(非退化假设) $\operatorname{dim}\left(T_{r_{i}(t)} W_{i}^{u} \cap T_{r_{i}(t)} W_{i+1}^{s}\right)=1, i=1,2, W_{3}^{s} \equiv W_{1}^{s}$.

（H3）(通有假设 2) 对 $i=1,2, e_{3}^{+} \equiv e_{1}^{+}$和 $e_{3}^{-} \equiv e_{1}^{-}$, 有

$$
\begin{aligned}
& \operatorname{span}\left(T_{r_{i}(t)} W_{i}^{u}, T_{r_{i}(t)} W_{i+1}^{s}, e_{i+1}^{+}\right)=\mathbb{R}^{m+n}, t \gg 1, \\
& \operatorname{span}\left(T_{r_{i}(t)} W_{i}^{u}, T_{r_{i}(t)} W_{i+1}^{s}, e_{i+1}^{-}\right)=\mathbb{R}^{m+n}, t \ll-1 .
\end{aligned}
$$

下面,在异宿环 $\Gamma$ 的邻域内建立一个局部坐标系, 它比文献 $[10,11]$ 中的要容易和简单 些.

设 $p_{i}$ 的邻域 $U_{i}$ 充分小. 总存在一个 $C^{r}$ 变换,使得系统 $(1.1)$ 在 $U_{i}$ 中有下面的形式:

$$
\begin{array}{ll}
\dot{x}=x\left[\lambda_{1}^{i}(\mu)+\cdots\right], & \dot{y}=y\left[-\rho_{1}^{i}(\mu)+\cdots\right], \\
\dot{u}=u\left[B_{1}^{i}(\mu)+\cdots\right], \quad \dot{v}=v\left[B_{2}^{i}(\mu)+\cdots\right],
\end{array}
$$

其中 $z=(x, y, u, v), x \in \mathbb{R}, y \in \mathbb{R}, u \in \mathbb{R}^{n-1}, v \in \mathbb{R}^{m-1}, \lambda_{1}^{i}(0)=\lambda_{1}^{i}, \rho_{1}^{i}(0)=\rho_{1}^{i}$, $\operatorname{Re}\left(\sigma\left(B_{1}^{i}(\mu)\right)\right)>\lambda_{0}^{i}, \operatorname{Re}\left(\sigma\left(B_{2}^{i}(\mu)\right)\right)<-\rho_{0}^{i}$.

(1.4) 式是 $C^{r-1}$ 的. 记 $A_{i}(t)=\mathrm{D} f\left(r_{i}(t)\right)$, 考虑线性系统及其伴随系统

$$
\begin{aligned}
& \dot{z}=A_{i}(t) z, \\
& \dot{\psi}=-A_{i}^{*}(t) \psi,
\end{aligned}
$$

此处, 符号 *表示转置. 下面, $T_{0}^{i}=T_{0}^{i}(\delta)$ 和 $T_{1}^{i}=T_{1}^{i}(\delta)$ 总表示满足 $r_{i}\left(-T_{1}^{i}\right)=(\delta, 0,0,0)$, $r_{i}\left(T_{0}^{i}\right)^{\prime}=(0, \delta, 0,0)$ 的时刻. 当假设 (H3) 成立时, 由文献 $[10,11]$ 知系统 (1.5) 有基解矩阵 $X_{i}(t)=\left(z_{1}^{i}(t), z_{0}^{i}(t), z_{2}^{i}(t), z_{3}^{i}(t)\right)$, 使得 $z_{1}^{i}(t) \in\left(T_{r_{i}(t)} W_{i}^{u}\right)^{c} \cap\left(T_{r_{i}(t)} W_{i+1}^{s}\right)^{c}, z_{0}^{i}(t)=$ $-\dot{r}_{i}(t) /\left|\dot{r}_{i}\left(T_{0}^{i}\right)\right| \in T_{r_{i}(t)} W_{i}^{u} \cap T_{r_{i}(t)} W_{i+1}^{s}, z_{2}^{i}(t)=\left(z_{21}^{i}, \cdots, z_{2, n-1}^{i}\right) \in T_{r_{i}(t)} W_{i}^{u u}, z_{3}^{i}(t)=\left(z_{31}^{i}\right.$, $\left.\cdots, z_{3, m-1}^{i}\right) \in T_{r_{i}(t)} \mathbb{W}_{i+1}^{s s}$,

$$
X_{i}\left(-T_{1}^{i}\right)=\left(\begin{array}{cccc}
\omega_{10}^{i} & \omega_{0}^{i} & 0 & \omega_{30}^{i} \\
\omega_{11}^{i} & 0 & 0 & \omega_{31}^{i} \\
\omega_{12}^{i} & 0 & I & \omega_{32}^{i} \\
0 & 0 & 0 & \omega_{33}^{i}
\end{array}\right), X_{i}\left(T_{0}^{i}\right)=\left(\begin{array}{cccc}
1 & 0 & \omega_{20}^{i} & 0 \\
0 & 1 & \omega_{21}^{i} & 0 \\
0 & 0 & \omega_{22}^{i} & 0 \\
\omega_{13}^{i} & 0 & \omega_{23}^{i} & I
\end{array}\right) \text {, }
$$

$\omega_{0}^{i}<0, \omega_{11}^{i} \neq 0, \operatorname{det} \omega_{22}^{i} \neq 0, \operatorname{det} \omega_{33}^{i} \neq 0$,

其中 $W_{i}^{n u}\left(W_{i}^{s s}\right)$ 为 $p_{i}$ 的强不稳定 (稳定) 流形. 而且, 当 $\delta$ 充分小 (即 $T_{0}^{i}$ 和 $T_{1}^{i}$ 充分大) 时, $\left|\omega_{j k}^{i}\left(\omega_{j j}^{i}\right)^{-1}\right| \ll 1, j=1,2,3, k \neq j$.

表 $\omega_{11}^{i}=\Delta^{i}\left|\omega_{11}^{i}\right|$, 当 $\Delta \equiv \Delta^{1} \Delta^{2}=1(-1)$ 时,称 $\Gamma$ 为非扭曲(扭曲)的. 


\section{Poincaré 映射}

首先,用 $\left(z_{1}^{i}(t), z_{2}^{i}(t), z_{3}^{i}(t)\right)$ 来定义 Poincare 截面。令

$$
\begin{gathered}
\Psi^{i *}(t)=X_{i}^{-1}(t)=\left(\psi_{1}^{i *}(t), \psi_{0}^{i *}(t), \psi_{2}^{i *}(t), \psi_{3}^{i *}(t)\right){ }^{*}, \\
S^{i}(t)=r_{i}(t)+z_{1}^{i}(t) n_{1}^{i}(t)+z_{2}^{i}(t) n_{2}^{i}(t)+z_{3}^{i}(t) n_{3}^{i}(t), \\
S_{0}^{i}=\left\{z=S^{i+1}\left(T_{0}^{i+1}\right):|z|<3 \delta / 2\right\}, S_{1}^{i}=\left\{z=S^{i}\left(-T_{1}^{i}\right):|z|<3 \delta / 2\right\}, \\
\psi_{2}^{i}(t)=\left(\psi_{21}^{i *}(t), \psi_{22}^{i *}(t), \cdots, \psi_{2, n-1}^{i *}(t)\right), \quad \psi_{3}^{i}(t)=\left(\psi_{31}^{i *}(t), \psi_{32}^{i *}(t), \cdots, \psi_{3, m-1}^{i *}(t)\right), \\
n_{2}^{i}(t)=\left(n_{21}^{i}(t), n_{22}^{i}(t), \cdots, n_{2, n-1}^{i}(t)\right)^{*}, \quad n_{3}^{i}(t)=\left(n_{31}^{i}(t), n_{32}^{i}(t), \cdots, n_{3, m-1}^{i}(t)\right)^{*} .
\end{gathered}
$$

再在 $\Gamma_{i}$ 的邻域内取坐标变换

$$
z(t)=S^{i}(t)=r_{i}(t)+\left(z_{1}^{i}(t), z_{2}^{i}(t), z_{3}^{i}(t)\right)\left(n_{1}^{i}(t), n_{2}^{i}(t), n_{3}^{i}(t)\right)^{*},
$$

$t \in\left[-T_{1}^{i}, T_{0}^{i}\right]$. 将 (2.1) 式代人 (1.1) 式,并利用 $\Psi^{i *}(t) X_{i}(t)=l$ 和

$$
f(z(t))=f\left(r_{i}(t)\right)+A_{i}(t)\left(z_{1}^{i}(t), z_{2}^{i}(t), z_{3}^{i}(t)\right)\left(n_{1}^{i}(t), n_{2}^{i}(t), n_{3}^{i}(t)\right)^{*}+\text { h.o.t. },
$$

可知系统 $(1.1)$ 变为 $C^{r-2}$ 系统

$$
\dot{n}_{j}^{i}(t)=\psi_{j}^{i *}(t) g\left(r_{i}, \mu\right)+\text { h.o.t. }, \quad j=1,2,3,
$$

其中 $i=1,2$. 系统 (2.2) 定义了由下式给出的映射 $P_{1}^{i}: S_{1}^{i} \rightarrow S_{0}^{i+1}$ :

$$
n_{j}^{i}\left(T_{0}^{i}\right)=n_{j}^{i}\left(-T_{1}^{i}\right)+M_{j}^{i} \mu+\text { h.o.t. },
$$

其中 $j=1,2,3, M_{2}^{i}=\left(M_{21}^{i}, M_{22}^{i}, \cdots, M_{2, n-1}^{i}\right), M_{3}^{i}=\left(M_{31}^{i}, M_{32}^{i}, \cdots, M_{3, m-1}^{i}\right)$,

$$
M_{1}^{i}=\int_{-T_{1}^{i}}^{T_{0}^{i}} \psi_{1}^{i *}(t) g_{\mu}\left(r_{i}, 0\right) \mathrm{d} t, \quad M_{j k}^{i}=\int_{-T_{1}^{i}}^{T_{0}^{i}} \psi_{j k}^{i *}(t) g_{\mu}\left(r_{i}, 0\right) \mathrm{d} t
$$

由系统 (1.4) 在 $U_{i}$ 中的特殊形式,易证

$$
M_{1}^{i}=\int_{-\infty}^{+\infty} \psi_{1}^{i *}(t) g_{\mu}\left(r_{i}, 0\right) \mathrm{d} t, \quad M_{j k}^{i}=\int_{-\infty}^{+\infty} \psi_{j k}^{i *}(t) g_{\mu}\left(r_{i}, 0\right) \mathrm{d} t .
$$

事实上, 当 $t \geqslant T_{0}^{i}\left(t \leqslant-T_{1}^{i}\right)$ 时, 仅仅 $g_{\mu}\left(r_{i}, 0\right)$ 的第 2(1) 个分量非零, 而 $\psi_{1}^{i}$ 和 $\psi_{j k}^{i}$ 的第 2(1) 个 分量为零 (详见文献 $[10]$ ).

在 $U_{i}$ 中, 流(2.1) 诱导了一个如下的映射 $P_{0}^{i}: S_{0}^{i} \rightarrow S_{1}^{i}$ :

$$
q_{0}^{i-1}:=\left(x_{0}^{i-1}, y_{0}^{i-1}, u_{0}^{i-1}, v_{0}^{i-1}\right)^{*} \mapsto\left(x_{1}^{i}, y_{1}^{i}, u_{1}^{i}, v_{1}^{i}\right)^{*}=: q_{1}^{i},
$$

其中 $u_{j}^{i}=\left(u_{j 1}^{i}, \cdots, u_{j, n-1}^{i}\right)^{*}, v_{j}^{i}=\left(v_{j 1}^{i}, \cdots, v_{j, m-1}^{i}\right)^{*}, q_{0}^{2}:=q_{0}^{0}$.

为简明计, 不妨设 $\rho_{1}^{i} \geqslant \lambda_{1}^{i}$. 记 $\tau^{i}$ 为从 $q_{0}^{i-1}$ 到 $q_{1}^{i}$ 经历的时间, $s_{i}=\exp \left\{-\lambda_{1}^{i}(\mu) \tau^{i}\right\}$ 为 Silnikov 时间. 略去高阶项, 可得(参见文献 $[10]$ )

$$
\begin{aligned}
& x_{0}^{i-1}=x_{1}^{i} s_{i}, \quad y_{1}^{i}=s_{i}^{\rho_{1}^{i}(\mu) / \lambda_{1}^{i}(\mu)} y_{0}^{i-1}, \\
& u_{0}^{i-1}=s_{i}^{B_{1}^{i}(\mu) / \lambda_{1}^{i}(\mu)} u_{1}^{i}, \quad v_{1}^{i}=s_{i}^{-B_{2}^{i}(\mu) / \lambda_{1}^{i}(\mu)} v_{0}^{i-1},
\end{aligned}
$$

其中 $x_{0}^{0}:=x_{0}^{2}, u_{0}^{0}:=u_{0}^{2}, v_{0}^{0}:=v_{0}^{2}$.

记 $P_{i}:=P_{1}^{i} P_{0}^{i}$, 则 $P_{i}$ 为系统 (1.1) 在异宿环 $\Gamma$ 的某个管状邻域内诱导的从 $S_{0}^{i}$ 到 $S_{0}^{i+1}$ 的 Poincaré 映射.

为得到 Poincaré 映射的表达式, 先要给出 $q_{0}^{i}, q_{1}^{i}$ 和它们的新坐标 $q_{0}^{i}\left(\bar{n}_{1}^{i}, \bar{n}_{2}^{i}, \bar{n}_{3}^{i}\right)$, 
$q_{1}^{i}\left(n_{1}^{i}\left(-T_{1}^{i}\right), n_{2}^{i}\left(-T_{1}^{i}\right), n_{3}^{i}\left(-T_{1}^{i}\right)\right)$ 间的关系. 利用

$$
\left(x_{0}^{i}, y_{0}^{i}, u_{0}^{i}, v_{0}^{i}\right)^{*}=r_{i}\left(T_{0}^{i}\right)+\sum_{j=1}^{3} z_{j}^{i}\left(T_{0}^{i}\right) \bar{n}_{j}^{i}, \quad\left(x_{1}^{i}, y_{1}^{i}, u_{1}^{i}, v_{1}^{i}\right)^{*}=S^{i}\left(-T_{1}^{i}\right)
$$

和 $X_{i}\left(-T_{\mathrm{I}}^{i}\right)$ 及 $X_{i}\left(T_{0}^{i}\right)$ 的表达式可得

$$
\begin{aligned}
& \bar{n}_{1}^{i}=x_{0}^{i}-\omega_{20}^{i}\left(\omega_{22}^{i}\right)^{-1} u_{0}^{i}, \quad \vec{n}_{2}^{i}=\left(\omega_{22}^{i}\right)^{-1} u_{0}^{i}, \\
& \bar{n}_{3}^{i}=v_{0}^{i}-\omega_{13}^{i} x_{0}^{i}+\left(\omega_{13}^{i} \omega_{20}^{i}-\omega_{23}^{i}\right)\left(\omega_{22}^{i}\right)^{-1} u_{0}^{i}, \\
& n_{1}^{i}\left(-T_{1}^{i}\right)=\left(\omega_{11}^{i}\right)^{-1}\left[y_{1}^{i}-\omega_{31}^{i}\left(\omega_{33}^{i}\right)^{-1} v_{1}^{i}\right], \\
& n_{2}^{i}\left(-T_{1}^{i}\right)=u_{1}^{i}-\omega_{12}^{i}\left(\omega_{11}^{i}\right)^{-1} y_{1}^{i}+\left(\omega_{12}^{i}\left(\omega_{11}^{i}\right)^{-1} \omega_{31}^{i}-\omega_{32}^{i}\right)\left(\omega_{33}^{i}\right)^{-1} v_{1}^{i}, \\
& n_{3}^{i}\left(-T_{1}^{i}\right)=\left(\omega_{33}^{i}\right)^{-1} v_{1}^{i}, \\
& y_{0}^{i}=\delta+\omega_{21}^{i} \bar{n}_{2}^{i} \approx \delta, x_{1}^{i}=\delta+\omega_{10}^{i} n_{1}^{i}\left(-T_{1}^{i}\right)+\omega_{30}^{i} n_{3}^{i}\left(-T_{1}^{i}\right) \approx \delta .
\end{aligned}
$$

因此, 由(2.3)、(2.4)和(2.8) (2.11) 式得到

$$
\begin{aligned}
& n_{1}^{i}\left(T_{0}^{i}\right)=\delta\left(\omega_{11}^{i}\right)^{-1} s_{i}^{\rho_{1}^{i}(\mu) / \lambda_{1}^{i}(\mu)}+M_{1}^{i} \mu+\text { h.o.t. }, \\
& n_{2}^{i}\left(T_{0}^{i}\right)=u_{1}^{i}-\delta\left(\omega_{12}^{i}\right)\left(\omega_{11}^{i}\right)^{-1} s_{i}^{\rho_{1}^{i}(\mu) / \lambda_{1}^{i}(\mu)}+M_{2}^{i} \mu+\text { h.o.t. }, \\
& n_{3}^{i}\left(T_{0}^{i}\right)=\left(\omega_{33}^{i}\right)^{-1} s_{i}^{-B_{2}^{i}(\mu) / \lambda_{1}^{i}(\mu)} v_{0}^{i-1}+M_{3}^{i} \mu+\text { h.o.t. }
\end{aligned}
$$

令 $G_{j}^{i}=n_{j}^{i}\left(T_{0}^{i}\right)-\bar{n}_{j}^{i}, j=1,2,3 ; i=1,2$, 则在 1 - 同宿, 1 - 周期和 1 - 异宿轨道与方程

$$
G_{l}^{i}(Q)=0, \quad G_{2}^{i}(Q)=0, \quad G_{3}^{i}(Q)=0 \quad(i=1,2)
$$

的满足 $s_{1}=0, s_{2}>0$ (或 $\left.s_{1}>0, s_{2}=0\right) ; s_{1}>0, s_{2}>0$ 和 $s_{1}=s_{2}=0$ 的解 $Q=\left(s_{1}, s_{2}, u_{1}^{1}, u_{1}^{2}, v_{0}^{1}\right.$, $\left.v_{0}^{2}\right)$ 之间分别存在着 1-1 对应.

\section{1-周期和 1-同宿轨道分支}

现在考虑 $\Gamma$ 附近的分支. 先设:

( I ) $\rho_{1}^{1}>\lambda_{1}^{1}, \rho_{1}^{1} / \lambda_{1}^{1}=\lambda_{1}^{2} / \rho_{1}^{2}=: \beta, \Delta=1, \operatorname{Rank}\left(M_{1}^{1}, M_{1}^{2}\right)=2$.

为保证在 $s_{2}=0$ 处的可微性, 在 $G_{j}^{i}$ 中作变换: $s_{1} \rightarrow s_{1}, s_{2} \rightarrow s_{2}^{\beta}$, 即令 $s_{2}=\exp \left\{-\rho_{1}^{2}(\mu) \tau^{2}\right\}$, 则 $G_{j}^{i}$ 变为

$$
\begin{aligned}
& G_{1}^{1}=\delta\left[\left(\omega_{11}^{1}\right)^{-1} s_{1}^{\beta}-s_{2}^{\beta}\right]+M_{1}^{1} \mu+\text { h.o.t., } \\
& G_{1}^{2}=\delta\left[\left(\omega_{11}^{2}\right)^{-1} s_{2}-s_{1}\right]+M_{1}^{2} \mu+\text { h.o.t., } \\
& G_{2}^{1}=u_{1}^{1}-\delta \omega_{12}^{1}\left(\omega_{11}^{1}\right)^{-1} s_{1}^{\beta}-\left(\omega_{22}^{1}\right)^{-1} s_{2}^{B_{1}^{2} / \rho_{1}^{2}} u_{1}^{2}+M_{2}^{1} \mu+\text { h.o.t. }, \\
& G_{2}^{2}=u_{1}^{2}-\delta \omega_{12}^{2}\left(\omega_{11}^{2}\right)^{-1} s_{2}-\left(\omega_{22}^{2}\right)^{-1} s_{1}^{B_{1}^{1} / \lambda_{1}^{1}} u_{1}^{1}+M_{2}^{2} \mu+\text { h.o.t., } \\
& G_{3}^{1}=\left(\omega_{33}^{1}\right)^{-1} s_{1}^{-B_{2}^{1} / \lambda} v_{0}^{1}-v_{0}^{1}+\delta \omega_{13}^{1} s_{2}^{\beta}+M_{3}^{1} \mu+\text { h.o.t. }, \\
& G_{3}^{2}=\left(\omega_{33}^{2}\right)^{-1} s_{2}^{-B_{2}^{2} / \rho_{1}^{2}} v_{0}^{1}-v_{0}^{2}+\delta \omega_{13}^{2} s_{1}+M_{3}^{2} \mu+\text { h.o.t. }
\end{aligned}
$$

考虑系统 (2.13) 的解. 易见当 $\delta, \mu$ 充分小时 $G_{2}^{i}=0$ 和 $G_{3}^{i}=0$ 总有解. 将其代人 $G_{1}^{i}=0$ $(i=1,2)$, 得到 


$$
\begin{gathered}
s_{2}=\omega_{11}^{2}\left(s_{1}-\delta^{-1} M_{1}^{2} \mu\right)+\text { h.o.t. }, \\
s_{1}^{\beta}+\delta^{-1} \omega_{11}^{1} M_{1}^{1} \mu+\text { h.o.t. }=\omega_{11}^{1}\left[\omega_{11}^{2}\left(s_{1}-\delta^{-1} M_{1}^{2} \mu+\text { h.o.t. }\right)\right]^{\beta} .
\end{gathered}
$$

假设 ( I ) 隐含着下述区域有定义:

$$
\begin{aligned}
& D_{i}:=\left\{\mu \in \mathbb{R}^{k}: M_{1}^{1} \mu>0,(-1)^{i} M_{1}^{2} \mu>0,0<|\mu| \ll 1\right\}, i=1,2, \\
& D_{j}:=\left\{\mu \in \mathbb{R}^{k}: M_{1}^{1} \mu<0,(-1)^{j} M_{1}^{2} \mu>0,0<|\mu| \ll 1\right\}, j=3,4 .
\end{aligned}
$$

记 (3.1) 式的左右端分别为 $L\left(s_{1}\right)$ 和 $N\left(s_{1}\right)$, 且

( II ) $\Delta^{1}=1, \Delta^{2}=1$.

命题 3.1 若假设 (I ) 和 (II) 成立, 则曲线 $y=L(s)$ 与曲线 $y=N(s)$ 在某点 $s_{1}\left(0<s_{1} \ll 1\right)$ 相切, 当且仅当 $\mu \in D_{1}, \omega_{11}^{1}\left(\omega_{11}^{2}\right)^{\beta}<1$ 且 $f(\mu)=f_{1}(\mu)$ 或者 $\mu \in D_{4}, \omega_{11}^{1}\left(\omega_{11}^{2}\right)^{\beta}>1$ 且 $f(\mu)=$ $f_{0}(\mu)$, 其中 $f(\mu)=-\delta^{-1} M_{1}^{2} \mu$,

$$
\begin{gathered}
f_{1}(\mu)=\left(\delta^{-1} M_{1}^{1} \mu\right)^{\frac{1}{\beta}}\left(\left(\omega_{11}^{2}\right)^{\frac{\beta}{1-\beta}}-\left(\omega_{11}^{1}\right)^{\frac{1}{\beta-1}}\right)^{\frac{\beta-1}{\beta}}+\text { h.o.t. }, \\
f_{0}(\mu)=-\left(-\delta^{-1} M_{1}^{1} \mu\right)^{\frac{1}{\beta}}\left(\left(\omega_{11}^{1}\right)^{\frac{1}{\beta-1}}-\left(\omega_{11}^{2}\right)^{\frac{\beta}{1-\beta}}\right)^{\frac{\beta-1}{\beta}}+\text { h.o.t. }
\end{gathered}
$$

证 $y=L(s)$ 与 $y=N(s)$ 在点 $s_{1}$ 相切, 当且仅当 $L\left(s_{1}\right)=N\left(s_{1}\right)$ 和 $L^{\prime}\left(s_{1}\right)=N^{\prime}\left(s_{1}\right)$, 即

$$
\begin{aligned}
& \omega_{11}^{1}\left(\omega_{11}^{2}\right)^{\beta}\left(s_{1}-\delta^{-1} M_{1}^{2} \mu+\text { h.o.t. }\right)^{\beta}=s_{1}^{\beta}+\delta^{-1} \omega_{11}^{1} M_{1}^{1} \mu+\text { h.o.t., } \\
& \omega_{11}^{1}\left(\omega_{11}^{2}\right)^{\beta}\left(s_{1}-\delta^{-1} M_{1}^{2} \mu+\text { h.o.t. }\right)^{\beta-1}=s_{1}^{\beta-1} .
\end{aligned}
$$

将 (3.3) 式代人 (3.2) 式, 可知上述方程有一个小的正解当且仅当 $\mu \in D_{1} \cup D_{4}$, 且此解为

$$
s_{1}=\left(\frac{-\omega_{11}^{1} M_{1}^{1} \mu}{M_{1}^{2} \mu}\right)^{\frac{1}{\beta-1}}+\text { h.o.t. }
$$

结合(3.3)和(3.4)式得到

$$
-\delta^{-1} M_{1}^{2} \mu=\left(\frac{-M_{1}^{1} \mu}{M_{1}^{2} \mu}\right)^{\frac{1}{\beta-1}}\left(\omega_{11}^{2}\right)^{\frac{\beta}{1-\beta}}-\left(\frac{-\omega_{11}^{1} M_{1}^{1} \mu}{M_{1}^{2} \mu}\right)^{\frac{1}{\beta-1}}+\text { h.o.t. }
$$

当 $\mu \in D_{1}$ 和 $\omega_{11}^{1}\left(\omega_{11}^{2}\right)^{\beta}<1$ 时, (3.5)式等价于 $f(\mu)=f_{1}(\mu)$. 假设 $\omega_{11}^{1}\left(\omega_{11}^{2}\right)^{\beta}<1$ 保证了 $\left(\omega_{11}^{2}\right)^{\frac{\beta}{1-\beta}}-\left(\omega_{11}^{1}\right)^{\frac{1}{\beta-1}}+$ h.o.t. $>0$.

当 $\mu \in D_{4}$ 和 $\omega_{11}^{1}\left(\omega_{11}^{2}\right)^{\beta}>1$ 时, (3.5) 式变为

$$
\left(\delta^{-1} M_{1}^{2} \mu\right)^{\frac{\beta}{\beta-1}}=\left(-\delta^{-1} M_{1}^{1} \mu\right)^{\frac{1}{\beta-1}}\left[\left(\omega_{11}^{1}\right)^{\frac{1}{\beta-1}}-\left(\omega_{11}^{2}\right)^{\frac{\beta}{1-\beta}}\right]+\text { h.o.t. },
$$

它等价于 $f(\mu)=f_{0}(\mu)$. 这里, 假设 $\omega_{11}^{1}\left(\omega_{11}^{2}\right)^{\beta}>1$ 相应于事实 $\left(\omega_{11}^{1}\right)^{\frac{1}{\beta-1}}-\left(\omega_{11}^{2}\right)^{\frac{\beta}{1-\beta}}+$ h.o.t. > 0 . 命题至此证毕.

由 $N(s)$ 的定义知 $N\left(s_{1}\right)=\omega_{11}^{1} s_{2}^{\beta}$, 且当 $\mu \in D_{2} \cup D_{4}$ 时 $N(s)$ 仅对 $s \geqslant \delta^{-1} M_{1}^{2} \mu+$ h.o.t. =: $f^{*}(\mu)$ 有定义. 由此易见, 对 $L(s)=N(s)$ 的任何解 $s_{1}$,

$$
s_{2}=\omega_{11}^{2}\left(s_{1}-\delta^{-1} M_{1}^{2} \mu\right)+\text { h.o.t. } \geqslant 0,
$$

且 $s_{2}=0$ 当且仅当 $s_{1}=f^{*}(\mu)$. 记

$$
f_{2}(\mu)=\left(\delta^{-1} M_{1}^{1} \mu\right)^{\frac{1}{\beta}}\left(\omega_{11}^{2}\right)^{-1}+\text { h.o.t. }, \quad f_{3}(\mu)=-\left(-\delta^{-1} \omega_{11}^{1} M_{1}^{1} \mu\right)^{\frac{1}{\beta}}+\text { h.o.t. },
$$


其中高阶项 h.o.t. 将在下面给定.

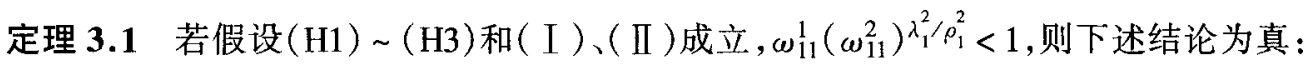

(1) 当 $\mu \in D_{1}$ 和 $f_{1}(\mu)<f(\mu)<f_{2}(\mu)$ 时, 系统 $(1.1)$ 在 $\Gamma$ 邻近恰有两个 1 -周期轨道.

(2) 当 $\mu \in D_{1}$ 和 $f(\mu)=f_{1}(\mu)$ 时,系统 (1.1) 在 $\Gamma$ 邻近有唯一的 2-重 1-周期轨道.

(3) 当 $\mu \in D_{1}$ 和 $f(\mu)=f_{2}(\mu)$ 时, 系统 (1.1) 在 $\Gamma$ 邻近恰有 1 个 1 - 周期轨道和 1 个 1 - 同 宿轨道。

(4) 当 $\mu \in D_{1}$ 和 $f(\mu)>f_{2}(\mu)$ (或 $f(\mu)<f_{1}(\mu)$ ) 时, 系统 (1.1) 在 $\Gamma$ 邻近恰有 1 个简单的 (或无) 1 - 周期轨道.

(5) 当 $\mu \in D_{2}$ 时,系统 (1.1) 在 $\Gamma$ 邻近总无 1 -周期轨道.

(6) 当 $\mu \in D_{3}$ 时,系统 (1.1) 在 $\Gamma$ 邻近总恰有 1 个简单的 1 - 周期轨道.

(7) 当 $\mu \in D_{4}$ 且 $f(\mu)>f_{3}(\mu)$ (或 $f(\mu)<f_{3}(\mu)$, 或 $f(\mu)=f_{3}(\mu)$ ) 时, 系统 $(1.1)$ 在 $\Gamma$ 邻 近恰有 1 个简单的 1 - 周期(或无 1 -周期,或恰有 1 个 1 -同宿) 轨道.

(8) 当 $M_{1}^{1} \mu=0$ (或 $\left.M_{1}^{2} \mu=0\right)$ 时,若 $M_{1}^{2} \mu<0$ (或 $\left.M_{1}^{1} \mu<0\right)$, 则系统 $(1.1)$ 在 $\Gamma$ 邻近恰有 1 个 简单的 1-周期轨道; 若 $M_{1}^{2} \mu>0$ (或 $M_{1}^{1} \mu>0$ ), 则在 $\Gamma$ 邻近无 1-周期(或 1-同宿)轨道; 若 $M_{1}^{2} \mu$ $=0$ (或 $\left.M_{1}^{1} \mu=0\right)$ ，则在 $\Gamma$ 邻近至多有 1 个 1 - 周期(或 1 -同宿) 轨道.

证 由(3.1) 和(3.6) 式, 只需考虑 $L(s)=N(s)$ 的非负解. 先设 $\mu \in D_{1}$.

令 $s=\alpha f(\mu), \omega=\omega_{11}^{1}\left(\omega_{11}^{2}\right)^{\beta}$, 则 $L(s)$ 和 $N(s)$ 变为下面的形式:

$L(\alpha):=f(\mu)^{\beta} \alpha^{\beta}+\delta^{-1} \omega_{11}^{1} M_{1}^{1} \mu+$ h.o.t. $, \quad N(\alpha):=\omega f(\mu)^{\beta}(\alpha+1)^{\beta}+$ h.o.t.

于是, 要求 $L(s)=N(s)$ 的小的正解, 只需求 $F\left(\alpha^{*}\right)=0$ 的正解 $\alpha^{*}$, 其中

$$
F(\alpha):=\alpha^{\beta}-\omega(\alpha+1)^{\beta}+\delta^{-1} \omega_{11}^{1} M_{1}^{1} \mu f(\mu)^{-\beta}+O(|\mu|) \text {. }
$$

由 $F^{\prime}(\alpha)=\beta\left[\alpha^{\beta-1}-\omega(\alpha+1)^{\beta-1}\right]+O(|\mu|)$ 知存在唯一的 $\alpha_{0}=1 /\left(\omega^{\frac{1}{1-\beta}}-1\right)+O(|\mu|)>0$, 使得 $F^{\prime}\left(\alpha_{0}\right)=0$. 因此,当 $\alpha>\alpha_{0}$ (或 $0<\alpha<\alpha_{0}$ ) 时, $F(\alpha)$ 是严格递增(或递减)的, $\alpha_{0}$ 是 $F(\alpha)$ 的唯一极小值.

再由当 $\omega<1$ 时 $\lim _{\alpha \rightarrow+\infty} \alpha^{\beta}-\omega(\alpha+1)^{\beta}=\lim _{\alpha \rightarrow+\infty} \alpha^{\beta}\left[1-\omega\left(1+\frac{1}{\alpha}\right)\right]=+\infty$ 知, 总存在 $\bar{\alpha}_{0}>\alpha_{0}>0$, 使得 $F\left(\bar{\alpha}_{0}\right)>0$.

另一方面,容易推知: $L(0)=N(0)$ 当且仅当

$$
f(\mu)=-\delta^{-1} M_{1}^{2} \mu=\left(\delta^{-1} M_{1}^{1} \mu\right)^{\frac{1}{\beta}}\left(\omega_{1 \mathrm{l}}^{2}\right)^{-1}+\text { h.o.t. }=: f_{2}(\mu) .
$$

由上述条件并考虑到 $\alpha_{0}(>0)$ 是 $F(\alpha)$ 的唯一极小值, 知 $F\left(\alpha_{0}\right)<0$. 由中值定理, 存在 $\alpha^{*} \in$ $\left(\alpha_{0}, \bar{\alpha}_{0}\right)$, 使得 $F\left(\alpha^{*}\right)=0$. 于是,结论 (3) 证毕.

现设 $f(\mu)=f_{1}(\mu)$. 由命题 3.1 的结论可得 $F\left(\alpha_{0}\right)=0$. 利用 $\alpha_{0}$ 是 $F(\alpha)$ 的唯一极小值 的事实,即可推出结论 (2).

从结论 (2) 和 (3) 的证明及下述事实: 当 $|\mu| \ll 1$ 时 $f_{2}(\mu)>f_{1}(\mu)$, 当 $f(\mu)<f_{2}(\mu)$ 时 $L(0)>N(0)$ (等价地, $F(0)>0$ ), 当 $f(\mu) \neq f_{1}(\mu)$ 时 $y=L(s)$ 与 $y=N(s)$ 不相切 (即 $F\left(\alpha_{0}\right) \neq$ $0)$, 即知结论 (1) 成立.

利用当 $f(\mu)>f_{2}(\mu)$ 时 $F(0)<0$ 和当 $f(\mu)<f_{1}(\mu)$ 时 $F(0)>0$ 及 $F\left(\alpha_{0}\right)>0$, 易证结论 (4). 
然后让 $\mu \in D_{3}$. 与上面类似, 总存在 $\bar{\alpha}_{0}>0$, 使得 $F\left(\bar{\alpha}_{0}\right)>0$. 由 $F(\alpha)$ 的定义, 知 $F(0)<$ 0 . 从中值定理知存在 $\alpha^{*} \in\left(0, \bar{\alpha}_{0}\right)$, 使得 $F\left(\alpha^{*}\right)=0$. 故结论 $(6)$ 成立.

再考虑 $\mu \in D_{2}$ 的情况. 此时总有

$$
N(s)=\omega\left(s-\delta^{-1} M_{1}^{2} \mu\right)^{\beta}+\text { h.o.t. }<\left(s-\delta^{-1} M_{1}^{2} \mu\right)^{\beta}<s^{\beta}<L(s),
$$

其隐含着结论 (5).

下设 $\mu \in D_{4}$. 现总有 $L^{\prime}(s)>N^{\prime}(s)$ 且 $N(s)$ 仅对 $s \geqslant f^{*}(\mu)=-f(\mu)+$ h.o.t. 有定义. 这说明 $L(s)=N(s)$ 当 $f(\mu)<f_{3}(\mu)$ 时无小的正解, 当 $f(\mu)=f_{3}(\mu)$ 时恰有一个解 $s_{1}=f^{*}(\mu)$ (从而, $\left.s_{2}=0\right)$. 另外, 当 $f(\mu)>f_{3}(\mu)$ 时, 令

$$
s=\alpha f^{*}(\mu), F_{1}(\alpha)=\alpha^{\beta}-\omega(\alpha-1)^{\beta}+\delta^{-1} \omega_{11}^{1} M_{1}^{1} \mu f^{*}(\mu)^{-\beta}+O(|\mu|),
$$

其中 $\alpha \geqslant 1$. 于是, $L\left(f^{*}(\mu)\right)<0=N\left(f^{*}(\mu)\right)$ 相应于 $F_{1}(1)<0$. 另一方面, 类似于情况 $\mu \in$ $D_{1}$, 可证总存在 $\bar{\alpha}_{0}>1$, 使得 $F_{1}\left(\bar{\alpha}_{0}\right)>0$, 从而总存在 $\alpha^{*} \in\left(1, \bar{\alpha}_{0}\right)$ 满足 $F_{1}\left(\alpha^{*}\right)=0$. 故结论 (7) 成立.

最后, 设 $M_{1}^{1} \mu=0$. 此时 $G_{1}^{1}=0$ 化为 $s_{1}=\left(\omega_{11}^{1}\right)^{1 / \beta} s_{2}+$ h.o.t., 而 $G_{1}^{2}=0$ 变成 $\left(1-\omega^{1 / \beta}\right) s_{2}=$ $-\delta^{-1} \omega_{11}^{2} M_{1}^{2} \mu+$ h.o.t., 于是结论 (8) 成立. 当 $M_{1}^{2} \mu=0$ 时的结论(8) 可类似地证明.

注意到当 $\mu \in D_{4}$ 和 $\left.\left.\omega_{11}^{1}\left(\omega_{11}^{2}\right)\right)^{2}\right)_{1}^{2} \rho_{1} \geqslant 1$ 时 $f_{3}(\mu)<f_{0}(\mu)$, 则下面的定理可用类似的方法证 明:

定理 3.2 若假设 $(\mathrm{H} 1) \sim(\mathrm{H} 3)$ 和 ( I )、( II ) 成立, $\omega_{11}^{1}\left(\omega_{11}^{2}\right) \lambda_{1}^{2} \rho_{1}^{2} \geqslant 1$, 则下述结论成立:

(1) 当 $\mu \in D_{1}$ 和 $f(\mu)<f_{2}(\mu)$ (或 $f(\mu)>f_{2}(\mu)$ ) 时,系统 (1.1) 在 $\Gamma$ 邻近恰有 1 个(或无) 1 - 周期轨道.

(2) 当 $\mu \in D_{1}$ 和 $f(\mu)=f_{2}(\mu)$ 时,系统 (1.1) 在 $\Gamma$ 邻近仅有 1 个 1 - 同宿轨道.

(3) 当 $\mu \in D_{2}$ 且 $\left.\omega_{11}^{1}\left(\omega_{11}^{2}\right)\right)_{1}^{2} / \rho_{1}^{2}>1$ (或 $\omega_{11}^{1}\left(\omega_{11}^{2}\right) \lambda_{1}^{2} / \rho_{1}^{2}=1$ ) 时,系统 (1.1) 在 $\Gamma$ 邻近恰有 1 个 简单的 (或无) 1 - 周期轨道.

(4) 当 $\mu \in D_{3}$ 时,系统 (1.1) 在 $\Gamma$ 邻近总无 1-周期轨道.

(5) 当 $\mu \in D_{4}, f(\mu)<f_{3}(\mu)$ 且 $\omega_{11}^{1}\left(\omega_{11}^{2}\right) \lambda_{1}^{2} / \rho_{1}^{2}>1$ (或 $\omega_{11}^{1}\left(\omega_{11}^{2}\right) \lambda_{1}^{2} / \rho_{1}^{2}=1$ ) 时, 系统 (1.1) 在 $\Gamma$ 邻近恰有 1 个(或无) 1 - 周期轨道.

(6) 当 $\mu \in D_{4}, \omega_{11}^{1}\left(\omega_{11}^{2}\right) \lambda_{1}^{2} / \rho_{1}^{2}>1, f(\mu)=f_{0}(\mu)$ (或 $\left.f(\mu)>f_{0}(\mu)\right)$ 时,系统(1.1) 在 $\Gamma$ 邻近 有唯一的 2-重 (或无) 1 - 周期轨道.

(7) 当 $\mu \in D_{4}, \omega_{11}^{1}\left(\omega_{11}^{2}\right) \lambda_{1}^{2} \rho_{1}^{2}>1, f_{3}(\mu)<f(\mu)<f_{0}(\mu)$ (或 $f(\mu)=f_{3}(\mu)$ ) 时, 系统 (1.1) 在 $\Gamma$ 邻近恰有两个 1 -周期 (或恰有 1 个 1 - 周期和 1 个 1 - 同宿) 轨道.

(8) 当 $\omega_{11}^{1}\left(\omega_{11}^{2}\right) \lambda_{1}^{2} / \rho_{1}^{2}>1, M_{1}^{1} \mu=0$ (或 $M_{1}^{2} \mu=0$ ) 时, 若 $M_{1}^{2} \mu>0$ (或 $M_{1}^{1} \mu>0$ ), 则系统 (1.1) 在 $\Gamma$ 邻近恰有 1 个简单的 1-周期轨道; 若 $M_{1}^{2} \mu<0$ (或 $M_{1}^{1} \mu<0$ ), 则在 $\Gamma$ 邻近无 1-周期或 1同宿轨道; 若 $M_{1}^{2} \mu=0$ (或 $M_{1}^{1} \mu=0$ ), 则在 $\Gamma$ 邻近至多有 1 个 1-周期或 1-同宿轨道.

注 3.1 在定理 3.1(3) (同样地, 在定理 3.2(2)) 中的 1-同宿轨道是同宿于鞍点 $p_{1}$ 的轨 道, 而在定理 3.1(7) (同样地, 在定理 3.2(7)) 中的 1-同宿轨道是同宿于鞍点 $p_{2}$ 的轨道.

以下记 $N(s)=\omega_{11}^{1}\left|\omega_{11}^{2}\right| \beta\left(\delta^{-1} M_{1}^{2} \mu-s+\text { h.o.t. }\right)^{\beta}$, 
( III ) $\Delta_{1}=-1, \Delta_{2}=-1$,

则 $N(s)$ 仅对 $0 \leqslant s \leqslant g^{*}(\mu):=\delta^{-1} M_{1}^{2} \mu+$ h. o.t. 有定义. 沿用命题 3.1 的证明, 并注意到 (3.4) 式在假设（III）下仍然有效,可得

命题 3.2 如果假设 (I) 和 (III) 成立, $M_{1}^{2} \mu \neq 0$, 则曲线 $y=L(s)$ 与曲线 $y=N(s)$ 相切于 点 $s_{1}$ 满足 $0<s_{1}<g^{*}(\mu) \ll 1$ 当且仅当 $\mu \in D_{2}$ 和 $g(\mu)=g_{1}(\mu)$, 其中

$$
g(\mu)=\delta^{-1} M_{1}^{2} \mu, g_{1}(\mu)=\left[\delta^{-1}\left|\omega_{11}^{1}\right| M_{1}^{1} \mu\right]^{\frac{1}{\beta}}\left[1+\left|\omega_{11}^{1}\right|^{\frac{1}{1-\beta}}\left|\omega_{11}^{2}\right|^{\frac{\beta}{1-\beta}}\right]^{\frac{\beta-1}{\beta}}+\text { h.o.t. }
$$

定理 3.3 假设 $(\mathrm{H} 1) \sim(\mathrm{H} 3)$ 和 $(\mathrm{I}) 、(\mathrm{III})$ 成立, $\mu \in D_{2}$, 并记 $g_{2}(\mu)=\left(\delta^{-1}\left|\omega_{11}^{1}\right| M_{1}^{1} \mu\right)^{\frac{1}{\beta}}+$ h.o.t. , $g_{3}(\mu)=\left(\delta^{-1} M_{1}^{1} \mu\right)^{\frac{1}{\beta}}\left|\omega_{11}^{2}\right|^{-1} \mid+$ h.o.t. 和 $\omega=\left|\omega_{11}^{1}\right|\left|\omega_{11}^{2}\right|^{\beta}$, 则下述结论成立:

(1) 当 $\omega<1$ 且 $g(\mu)>g_{1}(\mu)$ 或 $g(\mu)<g_{2}(\mu)$ 时,系统 (1.1) 在 $\Gamma$ 邻近无 1 - 周期轨道.

(2) 当 $\omega<1$ 且 $g_{2}(\mu)<g(\mu)<g_{3}(\mu)$ (或 $\left.g(\mu)=g_{2}(\mu)\right)$ 时, 系统 (1.1) 在 $\Gamma$ 邻近恰有 1 个简单的 1 -周期 (或 $1-$ 同宿) 轨道.

(3) 当 $\omega<1$ 且 $g_{3}(\mu)<g(\mu)<g_{1}(\mu)$ (或 $g(\mu)=g_{1}(\mu)$ ) 时, 系统(1.1) 在 $\Gamma$ 邻近恰有两 个(或唯一的 2-重) 1 - 周期轨道.

(4) 当 $\omega<1$ 且 $g(\mu)=g_{3}(\mu)$ 时, 系统 (1.1) 在 $\Gamma$ 邻近恰有 1 个 1 -周期轨道和 1 个 1 - 同宿 轨道,

(5) 当 $\omega>1$ 且 $g_{2}(\mu)<g(\mu)<g_{1}(\mu)$ (或 $g(\mu)=g_{1}(\mu)$ )时, 系统(1.1) 在 $\Gamma$ 邻近恰有两 个(或唯一的 2-重) 1 -周期轨道.

(6) 当 $\omega>1$ 且 $g_{3}(\mu)<g(\mu)<g_{2}(\mu)$ (或 $g(\mu)=g_{2}(\mu)$ ) 时, 系统 (1.1) 在 $\Gamma$ 邻近恰有 1 个简单的 1 -周期 (或 1 - 同宿) 轨道.

(7) 当 $\omega>1$ 且 $g(\mu)<g_{3}(\mu)$ 或 $g(\mu)>g_{1}(\mu)$ 时,系统(1.1) 在 $\Gamma$ 邻近无 1 - 周期轨道.

(8) 当 $\omega>1$ 且 $g(\mu)=g_{3}(\mu)$ 时,系统(1.1) 在 $\Gamma$ 邻近恰有 1 个 1 -同宿轨道.

(9) 当 $\omega=1$ 且 $g_{2}(\mu)<g(\mu)<g_{1}(\mu)$ (或 $\left.g(\mu)=g_{1}(\mu)\right)$ 时, 系统(1.1) 在 $\Gamma$ 邻近恰有两 个(或唯一的 2-重) 1 -周期轨道.

(10) 当 $\omega=1$ 且 $g(\mu)=g_{2}(\mu)$ 时,系统 (1.1) 在 $\Gamma$ 邻近恰有两个 1 - 同宿轨道,一个同宿于 $p_{1}$, 另一个同宿于 $p_{2}$.

(11) 当 $\omega=1$ 且 $g(\mu)<g_{2}(\mu)$ 或 $g(\mu)>g_{1}(\mu)$ 时, 系统 (1.1) 在 $\Gamma$ 邻近无 1-周期轨道.

证 首先注意到,分支方程 (3.1) 仅当 $s \leqslant g(\mu)$ 时有定义. 这意味着对 $L(s)=N(s)$ 的任 意解 $s_{1}$ 总有 $s_{2} \geqslant 0$. 同时, $L\left(g^{*}(\mu)\right)=N\left(g^{*}(\mu)\right)=\omega_{11}^{1} s_{2}^{\beta}=0$ 当且仅当 $g(\mu)=g_{2}(\mu)$, 而 $L\left(g^{*}(\mu)\right)>0($ 或 $<0)=N\left(g^{*}(\mu)\right)$ 当且仅当 $g(\mu)>g_{2}(\mu)$ (或 $\left.<g_{2}(\mu)\right)$.

其次,有 $N(0)=\omega_{11}^{1}\left|\omega_{11}^{2}\right|^{\beta}\left(\delta^{-1} M_{1}^{2} \mu\right)^{\beta}+$ h.o.t. $=\delta^{-1} \omega_{11}^{1} M_{1}^{1} \mu+$ h.o.t. $=L(0)<0$ 当且仅 当 $g(\mu)=g_{3}(\mu)$, 而 $N(0)<L(0)$ (或 $\left.>L(0)\right)$ 当且仅当 $g(\mu)>g_{3}(\mu)$ (或 $<g_{3}(\mu)$ ).

再次, 由 $g_{2}(\mu)$ 和 $g_{1}(\mu)$ 的定义, 易见 $g_{2}(\mu)<g_{1}(\mu)$, 且当 $\omega<1$ (或 $=1$ 或 $>1$ ) 和 $0<|\mu|$ $\ll 1$ 时,

$$
\begin{gathered}
g_{3}(\mu)-g_{1}(\mu)=\left[(\delta \omega)^{-1}\left|\omega_{11}^{1}\right| M_{1}^{1} \mu\right]^{\frac{1}{\beta}}\left[1-\left(\omega^{\frac{1}{\beta-1}}+1\right)^{\frac{\beta-1}{\beta}}\right]+\text { h.o.t. }<0, \\
g_{2}(\mu)-g_{3}(\mu)=\left(\delta^{-1}\left|\omega_{11}^{1}\right| M_{1}^{1} \mu\right)^{\frac{1}{\beta}}\left(1-\omega^{\frac{-1}{\beta}}\right)<0(\text { 或 }=0 \text { 或 }>0) .
\end{gathered}
$$


然后, 通过应用命题 3.2 和 $N^{\prime}(0)>0=L^{\prime}(0)$, 并沿用定理 3.1 的证明思想, 则所有的结论 均可不太困难地证得。

注 3.2 在上面的定理中, 出现于情况 (4) 和 (8)(或 (2)和 (6)) 中相应于 $g(\mu)=g_{3}(\mu)$ (或 $\left.g(\mu)=g_{2}(\mu)\right)$ 的 $1-$ 同宿轨道同宿于鞍点 $p_{1}$ (或 $p_{2}$ ).

注 3.3 当 $\Delta_{1}=-1, \Delta_{2}=-1$ 和 $\mu \in D_{4}$ 时,(3.1) 式仍对 $s_{1} \leqslant g(\mu)$ 有定义. 但由命题 3.2 知, 此时 $y=L(s)$ 和 $y=N(s)$ 无切点, 而且当 $0 \leqslant s \leqslant g(\mu)$ 时 $L(s)>0 \geqslant N(s)$. 由此推知此时 (1.1) 式在 $\Gamma$ 邻近无 1 -周期或 1-同宿轨道. 当 $\mu \in D_{1} \cup D_{3}$ 时有 $M_{1}^{2} \mu<0$. 此时 $G_{1}^{2}=0$ 意味 着当 $s_{1} \geqslant 0$ 和 $\mu$ 充分小时 $s_{2}=-\left|\omega_{11}^{2}\right|\left(s_{1}-\delta^{-1} M_{1}^{2} \mu\right)+$ h. o.t. $<0$, 这说明当 $\mu \in D_{1} \cup D_{3}$ 时 (1.1) 式在 $\Gamma$ 邻近无 1 - 周期或 1 - 同宿轨道. 通过比较 $s_{1}$ 和 $s_{2}$ 与 $|\mu|$ 的阶, 可推知当 $M_{1}^{1} \mu=0$, $M_{1}^{2} \mu \neq 0$ 和 $M_{1}^{1} \mu \neq 0, M_{1}^{2} \mu=0$ 时,(1.1) 式在 $\Gamma$ 邻近无 1-周期或 1-同宿轨道.

\section{4 平面异宿环的稳定性和分支}

在本节中, 设 $C^{r}$ 系统 $(1.1)$ 是 2 维的, $z=(x, y) \in \mathbb{R}^{2}, r \geqslant 5$, 并在 $\rho_{1}^{1} / \lambda_{1}^{1}=\lambda_{1}^{2} / \rho_{1}^{2}=: \beta$ 的情况 下考虑 $\Gamma$ 的稳定性和分支. 由文献 [4]知存在 $C^{r}$ 变换, 使在 $p_{i}$ 的邻域 $U_{i}$ 中 $(1.1)$ 式取下面的 形式:

$$
\dot{x}=\lambda_{1}^{i} x+x^{2} y R_{1}^{i}(x, y, \mu), \quad \dot{y}=-\rho_{1}^{i} y+x y^{2} R_{2}^{i}(x, y, \mu),
$$

其中 $R_{1}^{i}(x, y, \mu), R_{2}^{i}(x, y, \mu)(i=1,2)$ 为 $C^{r-3}$ 函数. 显见(4.1) 式已具有 (1.4)式的形式. 记

$$
\sigma_{i}=\exp \left\{\int_{-\infty}^{+\infty}\left(f_{x}+f_{y}\right)\left(r_{i}(t)\right) \mathrm{d} t\right\}, \quad \bar{\sigma}_{i}=\exp \left\{\int_{-T_{1}^{i}(\delta)}^{T_{0}^{i}(\delta)}\left(f_{x}+f_{y}\right)\left(r_{i}(t)\right) \mathrm{d} t\right\} .
$$

由下面命题 4.1 的证明易见积分 $\sigma_{i}$ 收玫当且仅当 $\rho_{1}^{i}=\lambda_{1}^{i}, i=1,2$. 当 $\rho_{1}^{i} \neq \lambda_{1}^{i}$ 时, 定义 $\sigma_{i}$ 为其 主值 $\sigma_{i}:=\lim _{\delta \rightarrow 0} \bar{\sigma}_{i}$.

命题 4.1 设 $\rho_{1}^{1} / \lambda_{1}^{1}=\lambda_{1}^{2} / \rho_{1}^{2}$, 则 $\sigma_{i}$ 收玫且 $\bar{\sigma}_{i}=\sigma_{i}$.

证 在 $U_{i}$ 中, 由 $(4.1)$ 式知 $f_{x}+f_{y}=\lambda_{1}^{i}-\rho_{1}^{i}+x y R^{i}(x, y, \mu)$, 其中 $R^{i}(x, y, \mu)$ 为 $C^{r-4}$ 函 数, 由此知

$$
\int_{-\infty}^{-T^{i}}+\int_{T_{0}^{i}}^{+\infty}\left(f_{x}+f_{y}\right)\left(r_{i}(t)\right) \mathrm{d} t=\int_{0}^{\delta}\left(1-\frac{\rho_{1}^{i}}{\lambda_{1}^{i}}\right) \frac{\mathrm{d} x}{x}+\int_{0}^{\delta}\left(\frac{\lambda_{1}^{i+1}}{\rho_{1}^{i+1}}-1\right) \frac{\mathrm{d} y}{y}=0,
$$

此即 $\bar{\sigma}_{i}=\sigma_{i}, i=1,2$.

因为发散量积分是任何 $C^{2}$ 变换的不变量 (参见文献 $[12,13]$ ), 故出现在 $\sigma_{i}$ 中的 $f$ 和 $r_{i}(t)$ 可视为它们在 $(1.1)$ 式中原来的形式.

命题 $4.2 \omega_{0}^{i}=-\lambda_{1}^{i} / \rho_{1}^{i+1}, \omega_{11}^{i}=\rho_{1}^{i+1} /\left(\lambda_{1}^{i} \sigma_{i}\right), i=1,2, \rho_{1}^{3}=: \rho_{1}^{1}$.

证 利用 $\dot{r}_{i}\left(T_{0}^{i}\right)=\left(0,-\rho_{1}^{i+1} \delta\right)$ 和 $\dot{r}_{i}\left(-T_{1}^{i}\right)=\left(\lambda_{1}^{i} \delta, 0\right)$, 可得

$$
z_{0}^{i}\left(-T_{1}^{i}\right)=-\dot{r}_{i}\left(-T_{1}^{i}\right) /\left|\dot{r}_{r}\left(T_{0}^{i}\right)\right|=\left(-\lambda_{1}^{i} / \rho_{1}^{i+1}, 0\right)=:\left(\omega_{0}^{i}, 0\right) .
$$

再由命题 4.1 和 Liouville 公式得到 $-\omega_{0}^{i} \omega_{11}^{i} \sigma_{i}=1$. 这就给出了 $\omega_{11}^{i}$ 的表达式.

因假设 $(\mathrm{H} 1) \sim(\mathrm{H} 3)$ 和 $($ II $)$ 对平面系统自动成立, 故由命题 4.2 和 $\omega_{11}^{1}\left(\omega_{11}^{2}\right)^{\beta}=\left(\rho_{1}^{1} / \lambda_{1}^{2}\right)^{\beta-1}$. $\left(\sigma_{1} \sigma_{2}^{\beta}\right)^{-1}$ 即知定理 3.1 和 3.2 可重述如下: 
定理 4.1 设 ( I ) 成立且 $m=n=1$, 则当 $\left(\lambda_{1}^{2} / \rho_{1}^{1}\right)^{\beta-1} \sigma_{1} \sigma_{2}^{\beta}>1$ (或 $\left.\left(\lambda_{1}^{2} / \rho_{1}^{1}\right)^{\beta-1} \sigma_{1} \sigma_{2}^{\beta} \leqslant 1\right)$ 时定 理 3.1 (或定理 3.2) 的结论 $(1) \sim(8)$ 为真.

注 4.1 由定理 4.1 , 只要 $\left(\lambda_{1}^{2} / \rho_{1}^{1}\right)^{\beta-1} \sigma_{1} \sigma_{2}^{\beta} \neq 1$, 在 $\rho_{1}^{1} / \lambda_{1}^{1}=\lambda_{1}^{2} / \rho_{1}^{2}$ 时 $\Gamma$ 仍然最多只能分支出 两个极限环. 如所周知, 1 个细同宿环当 $\sigma_{1} \neq 1$ 时至多能分支出两个极限环. 故上面的结论 是从细同宿环到细异宿环的一个自然的推广.

下面转向考虑 $\Gamma$ 当 $\rho_{1}^{1} / \lambda_{1}^{1}=\lambda_{1}^{2} / \rho_{1}^{2}$ 时的内稳定性. 由(2.4),(2.12)和 (2.13)式知, 当 $\mu=0$ 时 $P_{1}:=P_{1}^{1} P_{0}^{1}: S_{0}^{1} \rightarrow S_{0}^{2}$ 定义为 $n_{1}^{1}\left(T_{0}^{1}\right)=\delta^{1-\beta}\left(\omega_{11}^{1}\right)^{-1}\left(x_{0}^{2}\right)^{\beta}+$ h.o.t. 类似的, $P_{2}:=P_{1}^{2} P_{0}^{2}: S_{0}^{2} \rightarrow$ $S_{0}^{1}$ 定义为

$$
n_{1}^{2}\left(T_{0}^{2}\right)=\delta^{1-1 / \beta}\left(\omega_{11}^{2}\right)^{-1}\left(x_{0}^{1}\right)^{1 / \beta}+\text { h.o.t. 当 } \rho_{1}^{1} / \lambda_{1}^{1}=\lambda_{1}^{2} / \rho_{1}^{2}, \mu=0 .
$$

而且, $q_{0}^{1}=\left(x_{0}^{1}, \delta\right)=r_{1}\left(T_{0}^{1}\right)+z_{1}^{1}\left(T_{0}^{1}\right) \bar{n}_{1}^{1} \in S_{0}^{2}$ 既是 $P_{2}$ 的初始点, 同时又是 $P_{1}$ 的象, 故有 $\bar{n}_{1}^{1}=$ $n_{1}^{1}\left(T_{0}^{1}\right)$. 另一方面, 由 (2.6)式知当 $m=n=1$ 时有 $\bar{n}_{1}^{1}=x_{0}^{1}$. 这样, 当 $\mu=0$ 时 Poincaré 映射 $P:=P_{2} P_{1}$ 具有形式 $P\left(x_{0}^{2}\right)=\left(\omega_{11}^{1}\left(\omega_{11}^{2}\right)^{\beta}\right)^{-1 / \beta} x_{0}^{2}+$ h.o.t. 所以,下面的稳定性结论成立:

定理 4.2 设 $\rho_{1}^{1} / \lambda_{1}^{1}=\lambda_{1}^{2} / \rho_{1}^{2}=: \beta, m=n=1$, 则当 $\left(\lambda_{1}^{2} / \rho_{1}^{1}\right)^{\beta-1} \sigma_{1} \sigma_{2}^{\beta}<1$ (或 $>1$ ) 时 $\Gamma$ 是内稳 定(或不稳定)的.

注 4.2 定理 4.2 具较简洁的形式, 且完全用 $C^{2}$ 坐标变换的不变量来刻画判别准则, 而由 文献 $[3]$ 给出的准则更具构造性,且适用于多个鞍点的细异宿环. 另外, 在实际计算中, 积分 $\sigma_{i}$ 一般是发散的, 文献 [3] 给出的方法避免了积分的发散问题, 而应用定理 4.2 时则必须恰当地 选取 $\sigma_{i}$ 的主值.

\section{参考文献}

1 Reyn J W. Generation of limit cycles from seperatix polygons in the phase plane. In: Martini R, ed. Geometrocl Approaches to Differential Equations. Lect Notes in Math, Vol 810. New York: Springer-Verlag, 1980. $264 \sim 289$

2 Mourtada A. Degenerate and nontrivial hyperbolic polycycles with vertices. J Diff Equs, 1994, 113(1):68 83

3 Feng B. The stability of heteroclinic loop under the critical condition. Science in China, Ser A, 1991, 34(6): 673 684

4 Han M, Luo D, Zhu D. The uniqueness of limit cycles bifurcating from a singular closed orbit ( III ). Acta Math Sinica, 1992, 35(6): $673 \sim 684$

5 Battelli F, Lezzari C. Exponential dichotomies, heteroclinic orbits and Melinikov functions. J Diff Equs, 1990, 86(3): 342 366

6 Chow S N, Deng B, Terman D. The bifurcation of a homoclinic orbit from two heteroclinic orbits. SIAM J Math Anal, 1990, 21(2): $179 \sim 204$

7 Chow S N, Deng B, Terman D. The bifurcation of a homoclinic orbit from two heteroclinic orbits - a topological approach. Appl Anal, 1991, 42(2): $275 \sim 296$

8 Kokubu H. Homoclinic and heteroclinic bifurcations of vector fields. Japan J Appl Math, 1988, 5(4): 455 501

9 Zhu D. Exponential trichotomy and heteroclinic bifurcation. Nonl Anal TMA, 1997, 28(3): 547 557

10 Zhu D, Xia Z. Bifurcations of heteroclinic loops. Science in China, Ser A, 1998, 41(8): $837 \sim 848$

11 Zhu D. Problems in homoclinic bifurcation with higher dimensions. Acta Math Sinica, 1998, 14(3): $341 \sim 352$

12 Zhu D. Homoclinic bifurcation with codimension 3. Chin Ann Math, Ser B, 1994, 15(2): $205 \sim 216$

13 Zhu D. Invariants of coordinate Transformation. J East China Normal Univ (Nat Sci), 1998, (1):19 21 